\title{
Multilevel Authentication for Social Network
}

\author{
Dalian Wu Zhia Sheng", Isredza Rahmi A Hamid", Hannani Aman\# \\ \# Information Security Interest Group (ISIG), Faculty Computer Science \& Information Technology, \\ Universiti Tun Hussein Onn Malaysia, Malaysia \\ E-mail: angelsofmission@gmail.com,rahmi@uthm.edu.my,hanani@uthm.edu.my
}

\begin{abstract}
Nowadays, social network plays a vital role in communication. Normally user used social networks in daily activities. However, this left all online users susceptible to misuse and abuse. Recently, there has been a remarkable growth in number of hacking as well. Once the computer is online, anyone can have access to the network. Therefore, we proposed a secure social network site called SocialBook where users can post status, photos and connect with friends. This system is developed using PHP programming language and Iterative and Incremental Development methodology. The purpose of developing this system is to solve unsecured login accounts and lack of user authentication problem. This system applies the secret question procedure when the user wants to change their account password. Moreover, SocialBook use idle session timeout mechanism for additional security. When the user is idle for ten minutes, they will be logged out automatically. So, the user will be less worry about their account from being hacked and the identity impersonation by unauthorized user.
\end{abstract}

Keywords - Multilevel authentication, social network, security.

\section{INTRODUCTION}

Social network define way how we communicate. It is the core of communication which can be distinct as grouping of individuals into smaller and more specific groups, like between countries communities in a neighbourhood or neighbourhood subdivision [1]. The Internet is filled with millions of users who are looking to meet new friends. With the rise of online social network, users will use it to connect with others. Consequently, online users will be susceptible to misuse and abuse. Anyone who has access to the Internet can access the social network and carry out attacks such as stealing account information. Hence, security is a vital need in order to secure personal information on the Internet. There must be security measures to keep users and online credentials safe.

The information that has been published online is at risk of being compromised by others. In social network sites, there are many cases of accounts being hacked by someone else. The motives are either to steal valuable information from a user, as a sort of revenge, or targeted at famous users to shut them down out of jealousy. Therefore, it is important to keep the login information safe so that the third party user does not have the authority to steal our personal information.

In social networks, it is important to know who we are dealing with and whether the person is who they claimed to be. This is because the identity impersonation is security major concern in social media. Someone who is close with you in the social network may suddenly message to ask for your personal information such as bank account and password. In fact, the account was misused by someone else to obtain the confidential information. Hence, it is very important to authenticate the user before communicating with them. This project aims the specific goal to achieve several objectives:

i. to design multilevel authentication for social network website,

ii. to develop a social network website with multilevel authentication approach,

iii. to test the functionality of SocialBook system and user acceptance.

The SocialBook system will be focusing on how to keep the user information safe from the hackers. The security measures taken in this project will be a multilevel authentication. This is to ensure that each online social network account is only used by the intended user. The scope of the system can be divided into two that are user's scope and system's scope. Users of SocialBook need to answer secret question when they changed their account passwords. Other than that, the user can update their profiles, post messages in the social network and comment on other users' messages. For the system scope, the SocialBook is a web-based system that is built using Hypertext Preprocessor (PHP) language and Xampp as a web server. The system is able to keep users' accounts secured by activating the security questions when user wants to change the account 
password. There is an idle session timeout module which will $\log$ out the user automatically if it is left idle for ten minutes. User has to set the security question module during registration for authentication purposes.

The rest of the paper is organized as follows: Section II describes the related work on social networking and multilevel authentication. Section III presents the methodology used to develop the SocialBook system. Section IV discusses the result and discussion. Finally, Section V concludes the work and highlights a future research.

\section{THE MATERIAL AND METHOD/AlGORITHM}

This section discusses the literature review of social networking and multilevel authentication, background of social network, comparison of existing social network websites and technologies used.

\section{Social Networking}

Social Network Sites (SNS) have taken over as the platform where people communicate with each other. Social network sites is defined as web-based services that allow individuals to construct a public or semi-public profile within a bounded system, articulate a list of other users with whom they share a connection, and view or traverse their list of connections within the system [2]. Social networking websites have infiltrated daily lives by providing computeraided communications. The addition of functions and improvement of securities have drawn more users to use social network. Some of popular SNS websites are Twitter, Instagram and GooglePlus [3]. SNS rise rapidly because users just have to sign up for an account by providing basic information such as account name, password, and email.

SNS allows user to indulge in a cyber-environment by posting text and photos to friends who used the same SNS. Therefore, SNS allows users to present themselves in the cyber world and develop friendships with others. It is an alternative platform for introverts who looking for friend and afraid to communicate with others in real life. One of the factors that contribute to SNS's success is, it is free to use and easy to access anytime and anywhere as long as there is an Internet connection.

SNS allows people to communicate with each other based on trust. People share story and content with millions of friends online. Therefore, the information is susceptible to attacker with malicious intends to harm the user. People share date of birth, email addresses, house address, family relationships and pictures. This information is useful for hackers to perform attacks such as credit card fraud or identity fraud. Moreover, there is underground forum which sell personal information to criminals who want to obtain birth certificates, passwords or fake identity.

\section{Social Networking}

As the Internet continues to grow, it is vital for security protocols and procedures to always keep up to date [10][11]. Authentication plays an important part in security protocols. System without authentication, allows unauthorized users to access the systems and steal the data [4]. Due to increasing security concerns, multilevel authentication has gained momentum in the last few years [5].
Mostly in multifactor authentication, the first factor used is "something you know" such as username and password combination [12][14]. Users are required to login using valid username and password. The second factor is "something you have" which may include things that user own, such as mobile phone. Users are required to enter a short numerical code sent to mobile phone into the website for successful login. Last factor is "something you are", which refers to bio-metric details such as fingerprint, iris pattern and facial recognition. Everyone has unique and distinct fingerprint. Therefore, the attacker needs to have user's fingerprint in order to login successfully login. Nevertheless, the multilevel authentication could include a combination of these authentication methods.

\section{Existing System}

This section will discussed current social networking system such as Twitter, Instagram and Google Plus.

\section{1) Twitter}

Twitter is a free social networking website that allows registered users to post short posts on the timeline, known as tweets. There are many features on Twitter, such as users can follow and reply other users' tweets by using the Twitter application on smartphones or twitter.com website. Twitter's messages are limited to 140 characters and there is option to restrict delivery to circle of friends. Twitter also allows users to send messages via third party applications such as Tweetie, Twitterrific, and Feedalizr [6]. The advantages of Twitter are, it is available on every smartphone and user can follow everyone. Twitter also provides Short Message Service (SMS) notification to user. The disadvantage of Twitter is, it has many spam problems. User send spam messages by tweeting spam tweets. Moreover, it is only limited to 140 characters [7].

\section{2) Instagram [8]}

Instagram is an online mobile photo-sharing social network service that let user to take photos or videos and share them publicly or privately on the application. It also works based on the concept of having friends or followers. Instagram users can follow other users to see what they post, and then leave a "like" or comment in photo or video that they find interesting. User is required to get approval from other user before they can see their post depends on the privacy settings.

\section{3) Google Plus [9]}

Google Plus is a social network platform by Google. There are various features in Google Plus such as circles, hangouts and huddle. Instead of followers like Instagram, Google Plus has circles which are similar to category for your connections. User can share updates between circles of friend and family. Hangouts are similar to group video chats. It supports up to ten users at one time while huddle is used for group chats.

\section{4) SocialBook}

SocialBook is a social networking site that is developed to provide a better and safer social networking platform for daily communications between friends, family and outsider. It allows users to post status and describe what they are 
doing. User need to register a new account and request permissions from other user to be friends. Once accepted, friends are able to see the contents or status of their friends. User who is not in the friend's list is unable to see the content.

TABLE I

COMPARISON OF EXISTING SYSTEMS AND SOCIAL BOOK

\begin{tabular}{|l|c|c|c|c|}
\hline Features & Twitter & Instagram & $\begin{array}{l}\text { Google } \\
\text { Plus }\end{array}$ & SocialBook \\
\hline Login & $\sqrt{ }$ & $\sqrt{ }$ & $\sqrt{ }$ & $\sqrt{ }$ \\
\hline $\begin{array}{l}\text { Control who can } \\
\text { connect with you }\end{array}$ & $\mathrm{X}$ & $\sqrt{ }$ & $\mathrm{X}$ & $\sqrt{ }$ \\
\hline $\begin{array}{l}\text { Control whether } \\
\text { users can } \\
\text { message you }\end{array}$ & $\mathrm{X}$ & $\sqrt{ }$ & $\sqrt{ }$ & $\mathrm{X}$ \\
\hline $\begin{array}{l}\text { Choose who can } \\
\text { see your post }\end{array}$ & $\mathrm{X}$ & $\sqrt{ }$ & $\sqrt{ }$ & $\mathrm{X}$ \\
\hline Login alert & $\mathrm{X}$ & $\mathrm{X}$ & $\sqrt{ }$ & $\sqrt{ }$ \\
\hline Secret Question & $\mathrm{X}$ & $\mathrm{X}$ & $\mathrm{X}$ & $\sqrt{ }$ \\
\hline Session Timeout & $\mathrm{X}$ & $\mathrm{X}$ & $\mathrm{X}$ & \\
\hline
\end{tabular}

Table 1 shows the comparison between existing systems and the proposed system. In terms of privacy, Twitter has the least privacy controls. Twitter cannot control who connects with the user, who can message the user and who can see the user's post. On the other hand, Google Plus has better control of privacy. Google Plus also cannot control who connects with the user. But, it allows users to control who can message and see the post. In terms of security, Google Plus offered login alert which alerts the user when the account is logged in from a new device. On the other hand, Instagram has more control on user account. Yet, Instagram did not offer login alert function and secret questions for authentication purposes.

Our proposed social networking system differs than other system in such a way that, the SocialBook offer a multilevel authentication process. User needs to answer two secret questions when they changed the password account. If the SocialBook system is left idle for ten minutes, the user will be logged out automatically to prevent the account being accessed by unauthorized user.

This section discusses the methodology that has been used that is Iterative and Incremental Development and the procedures involved in the development of this system. Each phases of the methodology has its own objective which served as a guide to the system. Fig. 1 shows the Iterative and Incremental Development phases.

The advantages of Iterative Development cycle is selected because it provides feedback. This helps detect where a problem resides in each phase. This allows the system to response quickly to changes the errors. As there will be problems at every phases, it is identified and fixed and this process is repeated continuously. This will save time, cost and produces a flexible system which has an overall complete functioning system that has its issues fixed continuously to improve the system.

Fig. 2 shows the SocialBook system. The SocialBook system consists of users and admin. User is prompted with login menu. For new users, they are required to register an account before they can proceed to login the system. After registration, user is required to choose and answer two secret questions. Once the registration process is completed, user can login into the SocialBook system. User can post status on timeline and comment on friends' post. When user wants to change the account password, they need to answer the secret questions that have been set before. If the user idles for ten minutes, they will be logged out automatically and the user has to login the system again. Admin can view and ban all registered users and set the time for idle session timeout.

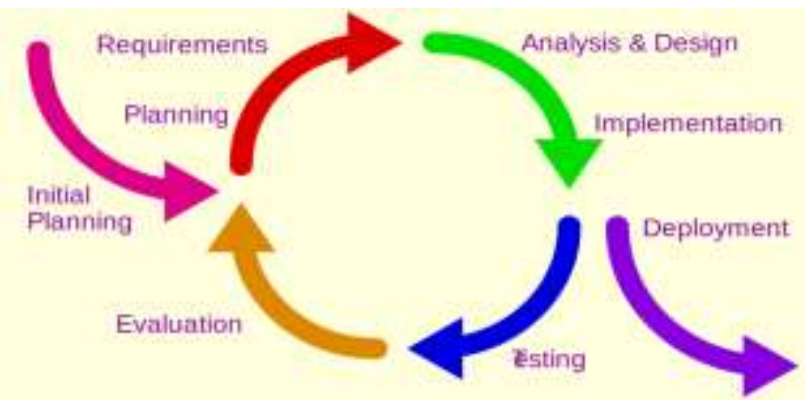

Fig.1 Phases of Iterative and Incremental Development [13]

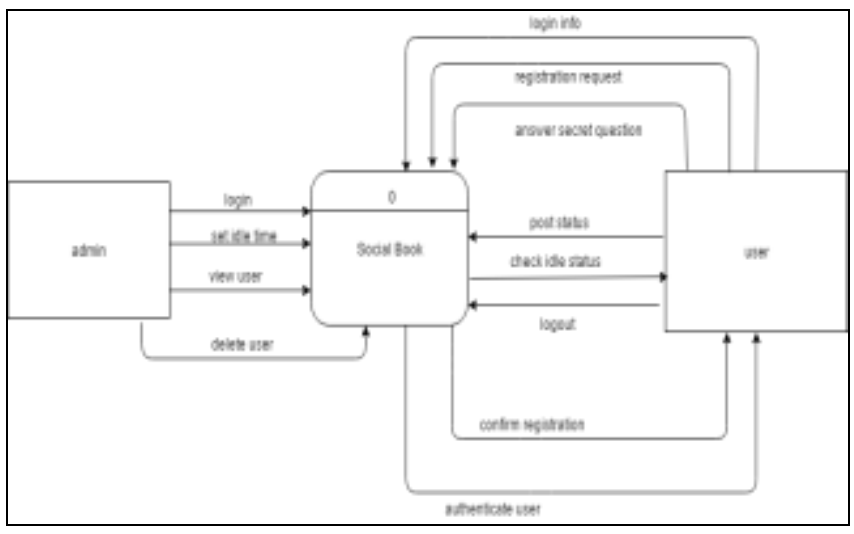

Fig.2 The SocialBook System

\section{RESULT AND DISCUSSION}

This section focuses on the implementation of the actual system. There are two main parts which are systems design and implementation and testing.

\section{A) System Design}

The interface of a system is designed to suit the needs and requirements of the users. The system interface is designed to be more users friendly and not too complex to ensure users can access the system more easily. This system is developed using PHP programming language and Iterative and Incremental Development methodology. The purpose of developing this system is to solve unsecured login accounts and lack user authentication problem.

\section{1) Login Page}

The connection between system and database was done using mysql_connect() function. Connection to the database allows the user to login, adding, updating and deleting information from the database.

Fig. 3 shows the login page of the SocialBook system. It has two options either sign up for new user or login function for current user. For registration, users need to provide first and last name, email, password, gender and date of birth. After signing up, users will proceed to upload a profile 
picture and choose and answer two secret questions as shown in Fig. 4 for multi authentication purposes.

The user account passwords are encrypted in the database using Advanced Encryption Standard with salt. The passwords are encrypted using the built-in Mysql AES_ENCRYPT function using a salt. Then, the user account passwords are decrypted using Advanced Encryption Standard (AES) decryption with the same salt.

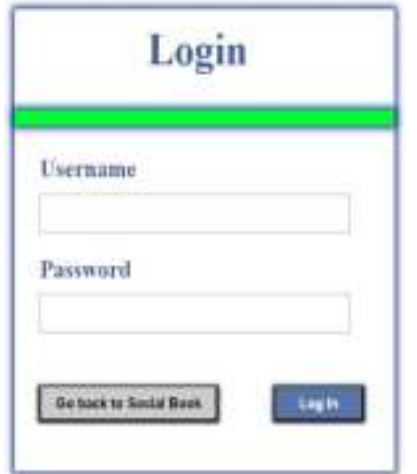

Fig.3 The SocialBook System Main Page

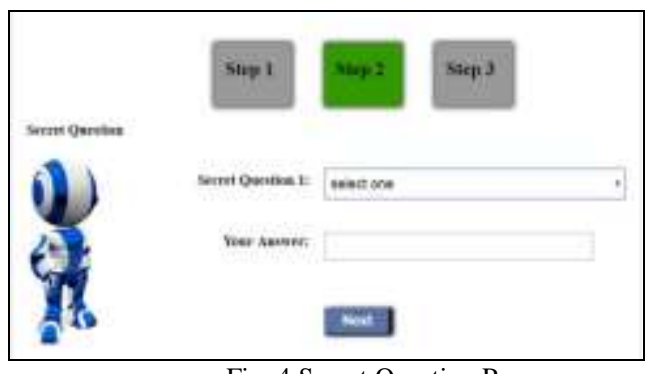

Fig. 4 Secret Question Page

\section{2) Main page}

Fig. 5 shows the main page of SocialBook system after user login into the system. User can post new status, upload image and delete their post on the timeline. The timeline will show all posts by friends as well. This system also applies the secret question procedure if the user wants to change their account password. The user has to answer the secret questions as the verification process.

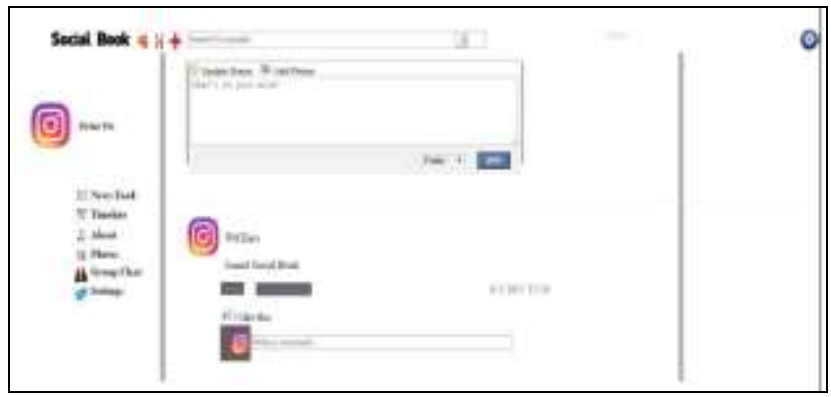

Fig. 5 SocialBook Main Page

\section{3) Session Timeout}

SocialBook use idle session timeout mechanism for additional security as in Fig. 6. When the user is idle for ten minutes, they will be logged out automatically. After the session starts, time in seconds is set that is required for session timeout. A new variable is declared that checks for inactive time. If inactive time is larger than the timeout set, user will be logged out from the system. So, the user will be less worry about their account from being hacked and the identity impersonation by unauthorized user.

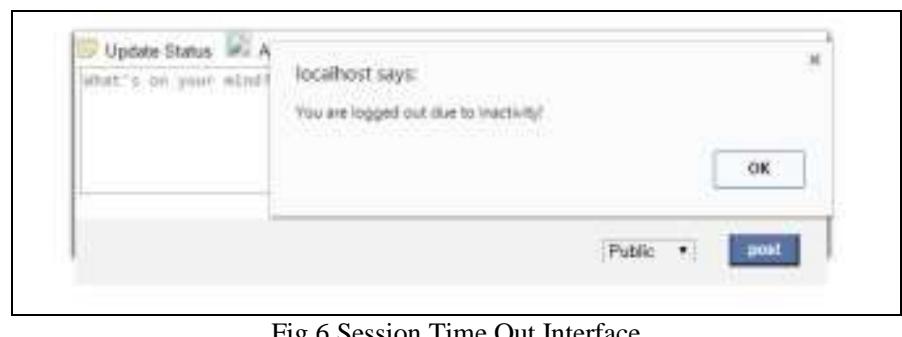

Fig.6 Session Time Out Interface

\section{B) Implementation and Testing}

Implementation is about the implementation of actual system based on the analysis and design that has been carried out. Testing is important to ensure that the SocialBook meets its requirements and users' needs. Therefore, the next section discusses the acceptance testing that was carried out with targeted users of SocialBook. Questionnaire is prepared and delivered to target user to test the proposed system. This is done to collect user's feedback, comments, bugs, and suggestions for improvement of SocialBook. The questionnaire is done on ten respondents representing five males and five females. Fig. 7 summarized the results of the questionnaire.

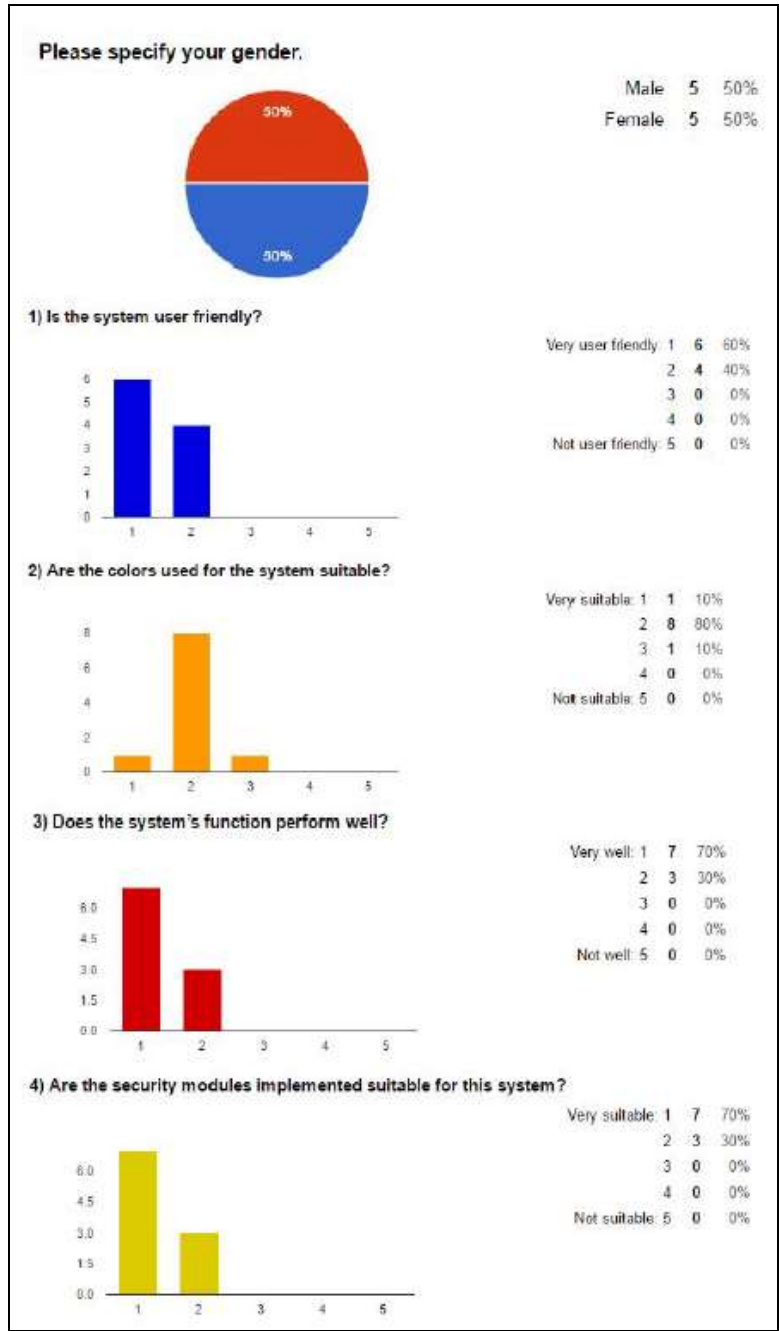

Fig.7 User Acceptance Testing 
Based on Fig. 7, approximately, $60 \%$ of respondents agree that the system is very user-friendly while $40 \%$ disagree. In terms of interface design, $10 \%$ respondents have the same opinion that the SocialBook system's colors were very suitable, $80 \%$ said it is suitable, while the rest agree the colour is moderate.

For SocialBook system function, $70 \%$ respondents said the system is functioning very well while $30 \%$ respondents thought it is functioning well. For the security modules, about $70 \%$ respondents agree that the security module is very sufficient while $30 \%$ of the respondent have the same opinion that the security module is suitable for SocialBook system.

\section{CONCLUSION}

SocialBook is a social network site built for communication between users. We study the existing systems such as Twitter, Instagram and Google Plus in order to develop SocialBook with improved security features. It provides multilevel authentication by using secret question and idle session timeout. In developing SocialBook, the Iterative and Incremental Development approach was used to develop the system. It has been tested by a list of test plans and test cases before ready for user.

The SocialBook system's limitation is lack of option to change the secret questions. User cannot change the secret question that they have selected during registration. In terms of functionality, the session timeout does not detect the mouse movements within the browser. Even there is an activity using the mouse, the user will be logged out from the system. So, user must perform interactive process in the SocialBook system such as clicking, posting or commenting within ten minutes.

Therefore, recommendation is needed to overcome the limitation of the system. We plan to add an option in the account settings page to have choices of secret questions that will be generated randomly. Moreover, the SocialBook system will be able to detect mouse movements in the browser to prevent session timeout by using javascript functions.

\section{ACKNOWLEDGEMENT}

The authors express appreciation to the Universiti Tun Hussein Onn Malaysia (UTHM). This research is supported by Short Term Grant vot number U653 and Gates IT Solution Sdn. Bhd. under its publication scheme.

\section{REFERENCES}

[1] P. K. Ryan, Social Networking. New York: rosen central, 2011.

[2] B.E. Nicole and D.M. Boyd, "Social Network Sites: Definition, History, and Scholarship," Journal of Computer-Mediated Communication, vol. 13, pp. 210-230, 2008.

[3] P. Kallas. (2016)Top 15 Most 2008Popular Social Networking Sites (and 10 Apps!). [Online]. Available: http://www.dreamgrow.com/top15-most-popular-social-networking-sites/

[4] M. A. Burrows. (1990) A logic of authentication. [Online]. Available: https://arxiv.org/ftp/arxiv/papers/1506/1506.03753.pdf

[5] B. Dickson. (2016) 5 authentication methods putting passwords to shame. [Online]. Available: http://thenextweb.com/insider/2016/03/31/5-technologies-will-flipworld-authentication-head/

[6] Findandtrace. (2015) Twitter Most downloaded and popular android apps in the world. [Online]. Available: http://www.findandtrace.com/telenews/twitter-most-downloaded-andpopular-android-apps-in-the-world/

[7] Sameer. (2014) Advantages and Disadvantages of Twitter. [Online] Available: http://www.muchtech.org/2014/04/advantages-anddisadvantages-of-twitter.html

[8] M. Sarah. (2015) Instagram review. [Online]. Available: https://www.cnet.com/products/instagram-android/review/

[9] C. J. P. Jaymar. (2013) Google+ (Android) review. [Online] Available:https://www.cnet.com/products/google-plusandroid/review/

[10] K. Burnham. (2015) How the top social networks compare on privacy -- in one handy chart. [Online]. Available: http://www.itworld.com/article/2915175/social-networking/how-thetop-social-networks-compare-on-privacy-in-one-handy-chart.html

[11] D. Glenn. (2012) The History of Social Media from 1978 - 2012 [Infographic]. [Online]. Available: http://www.webmasterview.com/2011/08/social-networking-history/

[12] C. Hoffman. (2015) The Best Two-Step Authentication: SMS, Autheticator Apps, and More. [Online]. Available: https://support.steampowered.com/kb_article.php?ref=4020-ALZM5519

[13] C. Larman, Agile and Iterative Development- A Manager's Guide. Pearson Education, 2004.

[14] N.S.K. Tanvi, "Multi-Dimensional and Multi-Level Authentication," International Journal of Computer Application (0975 - 8887), vol. 75(12), pp. 17-22, Aug. 2013. 